\title{
On the Role of Subjective Probabilities in Fire Risk Management Studies
}

\author{
FRANCIS NOONAN and ROBERT FITZGERALD \\ Fire Protection Engineering \\ Worcester Polytechnic Institute \\ Worcester, Massachusetts 01609, USA
}

\section{ABSTRACT}

If risk management studies in fire protection are to be implemented on a quantitative basis, it becomes necessary to estimate probability loss models for each risk management alternative. While actuarial data may exist for quantifying the risk of situations similar to the alternatives under review, there are usually enough differences or environmental uncertainties that direct substitution is considered to be inappropriate, and dependency on subjectively assessed probability loss models is a reality to be recognized. In our opinion, a common perception by practitioners in fire protection engineering is that subjective probabilities are "just guesses" and not to be used in any serious or rigorous fire protection risk management study. This paper points out that subjective probability assessments are valid representations of knowledge, and we seek to communicate guidelines from decision analysis for generating subjective probabilities within fire risk management studies. Keywords: Fire Risk Assessment, Subjective Probabilities, Probability Loss Models.

\section{INTRODUCTION}

Risk management for fire protection often reduces to a basic decision making problem. Given that certain assets have an identified fire risk exposure, then a choice may exist between accepting the current level of risk (i.e. the status quo alternative) or spending certain dollars in an effort to transfer, prevent or control the risk, and thereby reduce the likelihood of future loses. To make choices on a quantitative and systematic basis, it is necessary to perform quantitative risk assessment and generate probability loss models for each risk management alternative. The problem is that probability loss models are usually not known for the specific alternatives. While actuarial data may exist for quantifying the risk of situations similar to the alternatives under review, there are usually enough differences or environmental uncertainties that direct substitution is considered to be inappropriate. 
The fire protection engineering literature contains excellent surveys on fire risk modeling ([1], [2]). The literature reveals a variety of methods for modeling fire and assessing probability loss models ([3], [4] [5]). Despite the diversity that exists among these methods they share common ground in their dependence on subjective probabilities as inputs to the modeling process. A common perception by practitioners in fire protection engineering is that subjective probabilities are "just guesses" and not to be used in any serious or rigorous fire protection risk management study. This paper addresses the role of subjective probabilities in fire risk assessment. Our position is that, in the pragmatic world of risk management decision making, subjective probability is a valid representation of knowledge, and in the practice of fire protection engineering there will always be risk management studies which require the use of subjective probabilities. While criticism for risk assessment methods which fail to explain how model inputs should be obtained or criticism for studies which employ subjective probabilities in a casual manner is certainly well justified, the reaction of either doing nothing or falling back to performing only qualitative risk assessment is inappropriate. The discipline of decision analysis has made considerable progress in understanding how humans process information for making subjective probability assessments; guidelines for making subjective assessments more effectively have been identified. This paper reviews these results and interprets them for application in risk management studies. We hope that this improved understanding of subjective probability assessment will enable the fire protection community to practice quantitative risk assessment more frequently and more effectively.

\section{PROBABILITY VIEWPOINTS}

There are three viewpoints for probability. The objectivist viewpoint regards the probability of an event as the limit of the frequency with which the event occurs as the number of trials increases without limit. The objectivist considers the probability of an event to be a property of the event, and their concern is how to make an actuarial estimate of the event probability. The objectivist views the actuarial estimate as a measure of the empirical evidence. The subjectivist viewpoint, on the other hand regards the probability of an event as a number which represents a person's degree of belief that a statement about an event is true. The subjectivist considers a probability to be an attribute of the person making the assessment, and thereby disallows the notion of the assessment as being right or wrong. However, the subjectivist does require that encoded probabilities adhere to the basic axioms of probability and that evidence or knowledge be used in a rational and consistent manner. The mathematical viewpoint on probability works from assumptions of symmetry (on underlying elementary events) in conjunction with a rational or logical model in order to deduce the probability of higher level events.

To understand the conflict between the first two viewpoints, consider the probability of a fire next year in the building that you are in right now and, if there is a fire, consider the probability that the severity will exceed a specified level. There is a large volume of actuarial data on fire frequency and severity levels for various types of buildings. Also, there is a growing number of rational deterministic models for predicting fire growth over time. Can these different sources provide the information to answer relevant risk management questions? In general, one finds that as you seek data which provides good association with a specific building, the availability of 
objective data quickly dwindles, and may well vanish.

Suppose the building is a warehouse and one must evaluate the cost effectiveness of a sprinkler system installation. The evaluation requires estimates on the frequency of fire and the expected severity for the warehouse in question. For discussion purposes, focus on frequency. There is a good chance that you will be able to get an actuarial estimate on fire frequency for warehouses in general, but you may not be comfortable in assuming that this warehouse is typical with respect to frequency. Empirical evidence exists which shows that fire frequency in warehouses depends on a number of factors (e.g. type of building, type of materials stored), and, considering these factors, you feel that the warehouse in question presents greater than average risk. Consequently, you may wish to stratify the actuarial data on frequency, and focus on a data subgroup. It should be noted that frequency estimates require two types of actuarial data, fire incidence data and exposure level data. Therefore, we are really talking about being able to stratify two data bases. It is necessary to do this on each of the frequency relevant factors if one is to retrieve an actuarial estimate which is representative of the specific occupancy being assessed. However, the feasibility for stratification diminishes as the number of relevent factors increases because there is a progressively greater chance that either the data base of fire incidence or the one on exposure level will not contain the information that is necessary to perform stratification.

Suppose one is unable to stratify the actuarial data to obtain a subgroup which is representative of the warehouse in question. The pure objectivist will say that the frequency estimate is not available since representative actuarial data is not available. A subjectivist who understands the basic principles of decision analysis would recommend using an integration of the available objective (i.e. actuarial) and subjective data (i.e. expert opinion) with appropriate weights assigned to each data source. In this manner, an estimate of the frequency of a fire for the warehouse may be constructed. Later in this paper, some quidelines for doing this are provided.

When using any type of probabilistic analysis, subjective judgment and objective data are always linked. When actuarial statistics are used directly in risk assessment, one must make the judgment that the particular facility (or class of facilities) under study presents substantially the same experiment upon which the actuarial data was based. This is a subjective judgment.

Fire science models are deterministic and, by themselves, cannot yield probability loss models. Nevertheless, they also represent a source of objective data that can be linked to subjective judgement. For example, if a fire growth model predicts that a certain physical loss outcome will occur under a given set of conditions, then one can assess subjectively a degree of belief in how well the model represents the actual condition being assessed. Also, if the fire science model requires a set of input conditions, and these conditions are not known with certainty, then subjective (or actuarial) probabilities on the conditions can be introduced to generate a probability loss model.

The statement is sometimes made that, given enough time and effort, improvements in actuarial data bases and fire science models will make subjective assessments no longer necessary in risk management studies. This is misleading. Although advances will continue to improve the scope and accuracy of fire science models and actuarial data bases, changes also will continue in building designs and fire protection technology. For many risk 
management studies a gap will always persist, and subjective probability assessments will continue to play a key role. The process of using fire science models to generate probability loss models is illustrated in more detail later in the paper.

\section{GUIDELINES IN SUBJECTIVE PROBABILITY ASSESSMENT}

Although subjective probabilities are opinions and cannot be viewed as right or wrong, one still may judge the quality of the assessment. A consideration of decision analysis refers to normative and substantive goodness in assessments made by experts ([6]). Substantitive goodness refers to the amount of knowledge that the experts bring to the assessment process while normative goodness refers to the skill in translating the expert's knowledge base into probabilities. That is, does the expert fully utilize his or her knowledge and do they adhere to the basic axioms of probability?

All probabilities are ultimately conditional, and it should be reasonable that if substantive and normative goodness are to exist, then the event (and its qualifying conditions) should be defined clearly. This advice may seem trivial. However, recently we participated in an experiment for integrating subjective judgment and output from a fire science model to estimate the probability of compartment full room involvement. During the experiment, approximately half of the interaction time among the three experts was spent on trying to get agreement on what constitutes full room involvement.

As an analyst seeking to assure substantive goodness when eliciting subjective assessments from an expert, one must clearly understand the nature of the expert's knowledge base and construct an interface to access the expertise effectively. Procedurally, this means that one should avoid asking an expert to assess highly aggregate events. A fundamental principle of decision analysis is to exploit decomposition fully to more effectively solve a decision making problem [7]. In probability assessment, decomposing an event into subevents can provide the key to unlock the expert's knowledge base.

Event trees, fault trees and inference trees are established decomposition techniques. An event tree uses inductive logic to go from an initiating event to a set of possible final or complete events. For example, given that established burning has occurred in a building, one may wish to assess directly the probability of various loss levels. Alternatively, one may utilize an event tree to consider various scenarios for outcomes after established burning. Each scenario is a sequence of subevents that occur through time. The subevents address issues of fire growth, intervention response times and suppression effectiveness (see Figure 1). Presumably, the expert's knowledge base for assessing a probability loss model will be more fully utilized using the event tree rather than asking the expert to assess the likelihood for loss directly. 


\begin{tabular}{|c|c|c|c|c|}
\hline $\begin{array}{l}\text { ESTABLISHED } \\
\text { BURNING }\end{array}$ & $\begin{array}{l}\text { PRE-INTERVENTION } \\
\text { FIRE GROWTH } \\
\text { OUTCOMES }\end{array}$ & $\begin{array}{l}\text { DETECTION/ } \\
\text { SUPPRESSION } \\
\text { RESPONSE } \\
\text { TIME }\end{array}$ & $\begin{array}{l}\text { SUPPRESSION } \\
\text { EFFECTIVENESS }\end{array}$ & $\begin{array}{l}\text { SEVERITY } \\
\text { OUTCOME }(\$)\end{array}$ \\
\hline
\end{tabular}

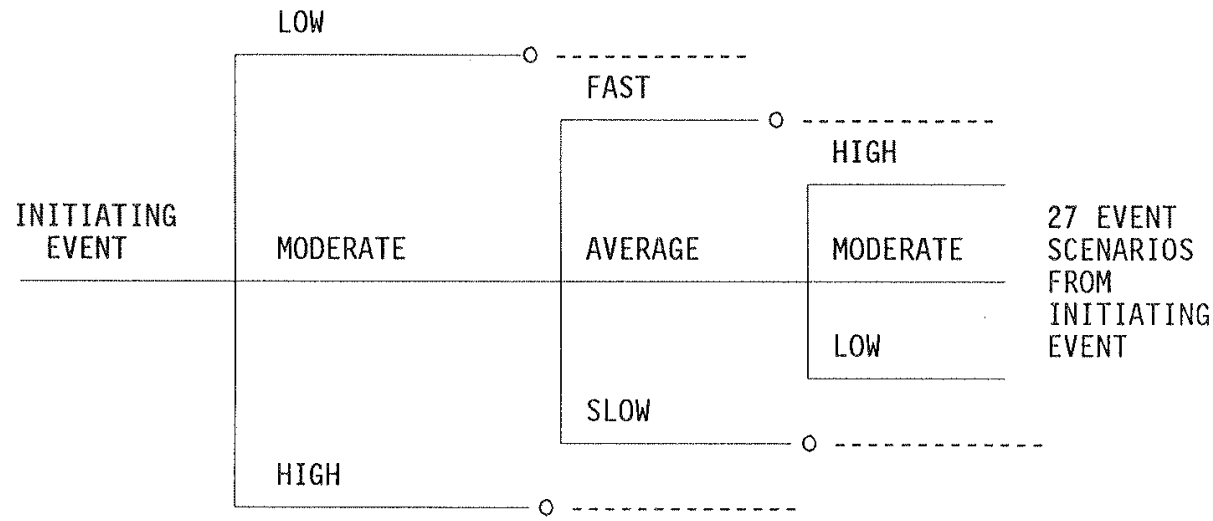

FIGURE 1 - EVENT TREE FOR MODELING SEVERITY (3 EVENT CATEGORIES FOR DECOMPOSITION)

A fault tree uses deductive logic to decompose the system by going from a complete or final event and branching backwards to consider all possible ways that the final event could occur. Inference trees allow one to explain an unobserved event (i.e. a hypothesis) in terms of observable events (i.e. data). Inference trees are commonly used in the insurance industry under the heading of risk scoring models [8], but their potential value in assisting quantitative risk assessment is probably not fully appreciated. Figure 2 illustrates one type of inference tree.

HYPOTHESIS WEIGHTS DATA

\begin{tabular}{|c|c|c|}
\hline & $20 \%$ & COMPARTMENT VOLUME \\
\hline & $15 \%$ & COMPARTMENT FLOOR AREA \\
\hline PROBABILITY & $15 \%$ & COMPARTMENT CEILING HT. \\
\hline FLASHOVER & $20 \%$ & BOUNDADY MATERTAI \\
\hline & $30 \%$ & AMT \& TYPE OF FUEL PKGS \\
\hline
\end{tabular}

FIGURE 2 - INFERENCE TREE (i.e. SCORING MODEL) FOR ASSESSING A COMPARTMENTS PROBABILITY FOR FULL ROOM INVOLVEMENT 
After decomposition one is confronted with a variety of probability assessment tasks. In each case, one is either dealing with a single event or with a random variable. To provide for normative goodness some guidelines are available. When assessing probabilities of single events (e.g. probability of ignition or probability of full room involvement), one should avoid asking experts to assess probabilities directly. The expert should use a reference event whose probability of occurrence is known (e.g. an actuarially known event), and then proceed to assess a relative probability. For assessing probability models of random variables (e.g. response time or severity of fire loss), the method of fractiles is recommended [9]. If there, is a random variable, $R V$ and the Probability $\{R V \leq x\}=p$, then $x$ is called the $p$-th fractile of RV. Under the method of fractiles, the expert assesses a discrete approximation to the random variable's probability distribution function by focusing on a few special fractile values, namely the $.01, .25, .5, .75$ and .99 fractiles. The assessor should not be asked to specify fractiles directly. The 0.5 fractile is determined by varying the $x$-value until indifference is assessed between the RV being above or below that value. The .01 and .99 fractiles are set by finding approximate lower and upper bounds. Research has shown that assessors tend to be overconfident or over conservative and not set the .01 and .99 fractiles as wide as possible. By making the assessor aware of this tendency, one can neutralize it to some extent. To continue, by conditioning the RV to be above the 0.5 fractile, one again can find an $x$-value which probabilistically divides that subrange evenly; the value is the 0.75 fractile. The 0.25 fractile can be assessed in a similar manner. The method of fractiles is similar to the quideline for assessing single event probabilities in that the procedure avoids assigning probability values directly. Figure 3 illustrates how the five fractile values can be smoothed to generate a cumulative probability distribution function for the RV in question.

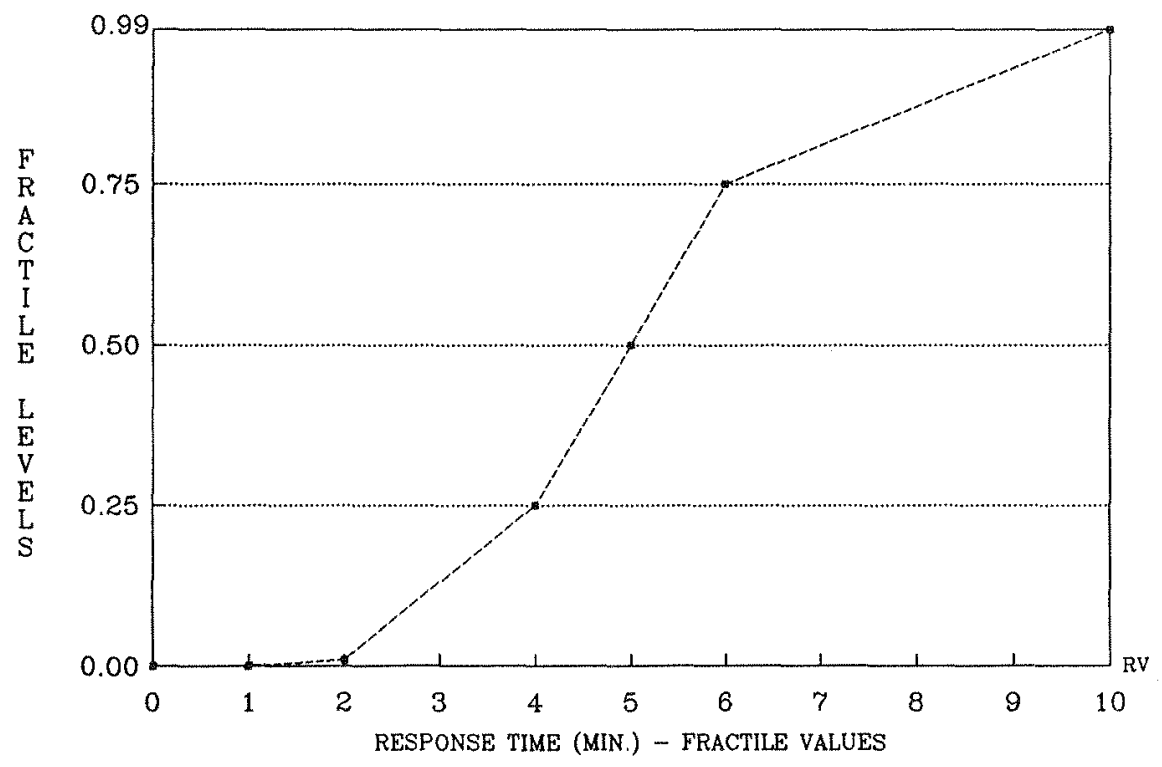

FIGURE 3 - CUMULATIVE PROBABILITY DISTRIBUTION ON RESPONSE TIME 
Experimental research in subjective assessment has shown bias on the part of human decision makers [10]. Humans display overconfidence in scientific knowledge, understate how human error can occur and have great difficulty in estimating rare events. To understand better the nature of human bias in performing subjective assessment tasks, we consider two types of information or data which experts utilize in applying subjective judgment ([11]). Singular information is case specific. It distinguishes the specific case from others in the same category. Distributional information is base rate data and concerns outcomes for a larger general class of situations. Subjective assessments require experts to draw upon both types of information. However, humans do not integrate this information well. Humans employ a number of heuristics to process information. Unfortunately, the heuristics can entail bias and detract from normative goodness. Under the representativeness heuristic, the probability of an outcome is approximated by the degree to which the outcome represents the essential features of the evidence. This can lead to overemphasizing singular information and ignoring prior or base rate statistics.

Using our previous example, suppose one needs to estimate the frequency of a fire in a warehouse which has no sprinkler system. Suppose that on an acturial basis the probability of a reportable fire during a year in any warehouse (i.e. sprinklered or not) is 1\%. A1so, $90 \%$ of a 11 reported warehouse fires had no sprinkler system and $75 \%$ of a 11 warehouses have no sprinkler systems. If $\mathrm{F}$ denotes the event of having a reportable warehouse fire and NS denotes the event of a warehouse having no sprinkler system then Bayes Rule for probabilities can be used to determine precisely the probability of a fire in a warehouse with no sprinkler system (i.e. the conditional event, $F / N S)$. Using $P(\cdot)$ to denote probability, then Bayes Rule gives

$P(F / N S)=\left[\frac{P(N S / F)}{P(N S)}\right] P(F)=\left[\frac{908}{75 \%}\right] \times 1 \%=1.2 \%$

The frequency of fires for all warehouses, $P(F)$, is an example of distributional or base rate information. while $P(N S / F)$, the evidentiary data linking reportable fires and the lack of sprinkler systems is an example of singular (i.e. more site specific) information. If actuarial probabilities on the events NS/F, NS and $F$ are available, then Bayes Rule is the mechanism for integrating the singular and distributional information and, thereby, estimating $P(F / N S)$. However, if $P(N S)$ is not known, then one must estimate $P(F / N S)$ subjectively.

The representativeness heuristic warns us that, as information processors, assessors may overemphasize the evidence linking fires to the absence of sprinklers and ignore the base rate data by estimating $P$ ( $F / N S$ ) to be much greater than the value of $1.2 \%$. In the extreme, the representativeness heuristic is called the Bayesian fallacy where one equates the likelihood of a fire for a certain type of occupancy to the likel ihood of finding that occupancy type, given there was a fire (i.e. $P(F / N S) \approx P(N S / F)$ ).

The anchoring heuristic can introduce bias in the opposite sense. Under the anchoring heuristic, assessments are formed by using a reference class for which outcome probabilities are known (i.e. distributional information) and, then adjusting the probabilistic value to include singular information. Bias 
occurs when conservatism and fear of uncertainty leads to underadjustment in utilizing singular information.

Another heuristic in subjective assessments is the availability heuristic which leads to approximating the probability of an outcome by the degree to which one can imagine the event. This can lead to bias toward retrievability of instance and improperly ignoring the longer term distributional information. For example, if a highly catastrophic fire recently occurred, one might overestimate the likelihood of such a fire occurring in a current risk management study. By understanding which type of bias which can exist, and by focusing on the two distinct types of information (i.e. singular and distributional), a risk analyst can do a better job in bringing normative goodness to the process of subjective probability assessment. More practical guidelines follow.

First, the concept of substantive goodness for risk assessment should be interpreted in the pragmatic world of risk management decision making. Substantive goodness is relative; it is maximized when one uses all information that is available within the time and monetary constraints of the particular risk management study. If there is interest in making a greater conversion of qualitative risk assessment studies over to quantitative risk assessment, it may be more reasonable to focus first on low cost assessment methodology which has a greater chance for adoption. In any event, fire risk analysts should recognize that one could have two widely differing risk assessment methodologies, each of which may offer a similar degree of substantive goodness even though a commensurate difference in the time and cost requirements for the two methods may exist. Whatever methodology is uded, an awareness for the two types of knowledge or information available to the probability assessment task is necessary. Distributional information will include actuarial data and usually can be formatted in term of probability loss model parameters. Singular information concerns site specific observations or data for the particular subject of the risk management study. It is less likely that singular information can be formatted directly as probability loss model parameters.

When assessing the parameter of a probability loss model subjectively, one must first select a reference class for which actuarial or fire science data is available. While it is desirable to relate the reference class as closely as possible to the specific case under investigation, tradeoffs must be made between the similarity of the reference class to the specific assessment task and the quality of the actuarial or fire science data available for the reference class. Using an actuarial risk parameter estimate as an anchor, one must focus on the singular information that is available (i.e. information which distinguishes the specific case from the general characteristics of the reference class), and subjectively assess how much the actuarial risk parameter should be adjusted (i.e. subjective assessment = (adjustment factor) $\times$ (actuarial risk parameter)). The adjustment factor can be determined by a direct subjective judgment, or it can be enhanced by exploiting decomposition and the use of scoring models (i.e. inference trees). For the latter case, one can examine the available singular information and identify a set of observable factors $\left(F_{i}, i=1, \ldots I\right)$ which can be scored for both the specific situation and the reference class. The factors chosen should be explanatory with respect to the value of the risk parameter. The relative importance of the factors for predicting the risk parameter can be assessed subjectively, and then calibrated to conform to the reference class. That is, 
$\underset{\text { Assessment }}{\text { Aubjective }}=\left\{\sum_{i=1}^{I} w_{i} F_{i}\right\} X\{$ Actuarial Risk Parameter $\}$

where $\sum_{i=1}^{I} w_{i} F_{i}^{*}=1$ and $\left(F_{i}^{*}, i=1, \cdots, I\right)$ denotes the factors scored for the reference class.

When the source of objective data is not an actuarial probability but output from a fire science model, the adjustment process is different. The fire science model will simulate the physical conditions for some stage of a fire process over time (e.g. a compartment's upper layer temperature before intervention, $T$ ). If one can define the critical event( $s)$ in terms of threshold conditions on the physical variables being modeled (e.g. FL denotes flashover which occurs when $T \geq T^{*}$ ), then one can use the fire science model to estimate the probability of flashover (i.e. $P(F L)$ ). The human expert makes a subjective judgment on how well the fire science model represents reality. If the representation is not perfect, then the model either understates or overstates the physical variables and the expert must come to terms with the nature of this approximation. When $T \geq T^{*}$ and you believe that the model understates $T$ then $P(F L)=1$. However, if you believe that the model overstates $T$, then one must integrate that opinion subjectively with the difference, $T-T^{*}$, in order to estimate a value for $P(F L)$ between $(0,1)$. Conversely, if $T_{<} T^{*}$ and you believe the model understates $T$, the expert again must integrate the objective knowledge, $T^{*}-T$, with the subjective judgment on the model's quality in order to generate an estimate on $P(F L)$.

Uncertainty can be appended to fire science models in a second way. If the model requires specifying a set of input conditions (denoted by I) and these conditions are not known with certainty, one then must consider a probability model where $\left(I_{n}, n=1, \ldots, N\right)$ denotes the set of $N$ possible input conditions. For each condition, one has an objective or subjective probability, $p_{n}$. The final result, $P(F L)$ can be given by $\sum_{n=1}^{N} P\left(F L_{n}\right) P_{n}$.

The discipline of decision analysis has made considerable progress in understanding how humans process information for making subjective probability assessments. Guidelines for making more effective subjective assessments have been presented with the hope that improved understanding of subjective probability assessments will enable the fire protection community to use this tool more effectively in research and practice. 


\section{REFERENCES}

1. Ling, W-C.T., Williamson, R.B., "Modeling Fire Spread Through Probabilistic Networks", Fire Safety Journal, 9: 287-300, 1985.

2. Elms, D.G., "Modeling Fire Spread in Buildings", Fire Technology, 20: 1, $11-19,1984$.

3. Yeh, Kwan-Nan "Ignition Risk Analysis - Cigarette Ignition of Upholstered Furniture", Fire Technology, 21: 2, 105-121, 1985.

4. CONCAWE's Ad-Hoc Risk Assessment Group, "Methodologies for Hazard Analysis and Risk Assessment in the Petroleum Refining and Storage Industry", Fire Technology, 20: 3, 23-56, 1984.

5. Watts, J.M., "Dealing with Uncertainty: Some Applications in Fire Protection Engineering", Fire Safety Journal, 11: 127-134, 1986.

6. Winkler, R.L., Murphy, A.H., "Good Probability Assessors", Journal of Applied Meteorology, 7: 751-758, 1968.

7. Von Winterfeldt, Detlof, Edwards Ward, Decision Analysis and Behavioral Research, pp. 45-62, Cambridge University Press, Cambridge, Massachusetts, 1986.

8. Kaiser, J. "Experiences of the Gretener Method", Fire Safety Journal, 2: $213-222,1980$.

9. P. Vatter, Bradley, S.P., Sherwood C.F. Jr. and Jackson, B.B, Quantitative Methods in Management, pp. 165-183, Richard D. Irwin, Inc., Homewood, I17inois 1978 .

10. Tversky A. and Kahneman D., "Uncertainty: Heuristics and Biases", Science, 185: 1124-1131, 1974.

11. Kahneman, D., Slovic, P. and Tversky, A., Judgement Under Uncertainty, pp. 414-421, Cambridge University Press, Cambridge, Massachusetts, 1982. 